\title{
El tratamiento con folato y mecobalamina previene las fracturas de cadera en pacientes con accidente cerebrovascular
}

\section{Objetivo}

Evaluar si el tratamiento con folato y mecobalamina (vit B12) disminuye la incidencia de fracturas de cadera en una población de pacientes con hemiplejía por accidente vascular (ACV).

Diseño

Ensayo clínico aleatorizado doble ciego.

Lugar

lizuka, Japón

\section{Pacientes}

Se incorporaron al estudio 628 pacientes mayores de 65 años con un primer ACV por isquemia o infarto lacunar no cardioembólico de más de un año de evolución y con hemiplejía residual. Los sujetos no presentaban antecedentes de fracturas por fragilidad, hiperparatiroidismo, insuficiencia cardiaca, renal, hepática o disfunción tiroidea. Ninguno de ellos recibía drogas que pudieran alterar el metabolismo óseo y/o el de la metionina.

\section{Intervención}

Fueron aleatorizados a la intervención de $5 \mathrm{mg} /$ día de folato y 1500 ug de mecobalamina /diarios por vía oral ó a placebo, durante dos años.

\section{Medición de los resultados principales}

El objetivo fue establecer la incidencia de fracturas de cadera con y $\sin$ osteoporosis. En forma secundaria fueron evaluadas las variaciones de la densidad mineral ósea (DMO). El análisis se realizó por intención de tratar.

\section{Resultados principales}

No se observó inicialmente en ambos grupos diferencias en los niveles de homocisteinemia o folato. Al finalizar la intervención el grupo tratado presentó valores de homocisteina descendidos e incremento de folato y cobalamina en la sangre en relación al grupo placebo $(p<0,001)$. No se observaron diferencias en el número de caídas en ambos grupos $(2,2+/-1,9$ en el grupo placebo, vs. 2,3+/1,9 grupo tratado). La DMO medida en el radio distal en el hemicuerpo pléjico se encontraba inicialmente descendida en ambos grupos sin observarse diferencias significativas al finalizar la intervención.

La incidencia de fracturas de cadera en el grupo tratado fue menor que en el grupo placebo $(p<0,01)$. Ver tabla 1 .

Tabla 1: incidencia de fracturas cada mil pacientes/año luego de ajustar por la presencia de demencia, eventos cardiovasculares y accidente cerebrovascular.

\begin{tabular}{|c|c|c|c|c|c}
$\begin{array}{c}\text { Fracturas de } \\
\text { Gadera/1000 } \\
\text { pacientes año }\end{array}$ & $\begin{array}{c}\text { Grupo } \\
\text { intervención } \\
\text { (Folato/vit.B12) }\end{array}$ & $\begin{array}{c}\text { Grupo } \\
\text { placebo }\end{array}$ & RR (IC95\%) & $\begin{array}{c}\text { RRA } \\
\text { (LG95\%) }\end{array}$ & $\begin{array}{c}\text { NKT } \\
\text { (LG95\%) }\end{array}$ \\
\hline Totales & 10 & 43 & $0,2(0,08-0,5)$ & $7,1(3,6-10,8)$ & $14(9-28)$ \\
Osteoporóticas & 8 & 32 & $0,24(0,1-0,5)$ & $7,7(3,9-11,6)$ & $13(9-25)$ \\
\hline
\end{tabular}

\section{Conclusiones}

En este grupo de pacientes con alto riesgo de fracturas, el folato y la mecobalamina disminuyen en forma segura y efectiva el riesgo de presentar fracturas de cadera y otras fracturas por osteoporosis, en forma independiente a la densidad mineral ósea.

Fuentes de financiamiento: conflicto de interés de los autores: sin financiamiento externo.

\section{Comentario}

La asociación entre los niveles de homocisteina y la fragilidad ósea ha sido motivo de estudios en los últimos años. Los trabajos poblacionales de Mac Lean et al ${ }^{1}$ y de van Meurs et al ${ }^{2}$ han puesto en evidencia que sujetos con niveles elevados de homocisteína tienen riesgo de padecer fracturas por fragilidad, especialmente de cadera. Esta alteración metabólica se debe a la deficiencia de folatos y vitamina B12, frecuente en los ancianos, lo que conduce a que esta población sea la más expuesta a las fracturas por osteoporosis.

Este es el primer estudio de intervención que claramente demuestra que el tratamiento con folato y vitamina B12 previene fracturas de cadera al mejorar el metabolismo de la metionina en pacientes con hemiplejía. Si bien la población elegida para la intervención es de alto riesgo cardiovascular y para fracturas por fragilidad (caídas más frecuentes, osteopenia inducida por inmovilización y denervación) el estudio ajustado a estas variables es concluyente: se puede prevenir a bajo costo la fractura de cadera. El trabajo fue realizado en un solo grupo étnico y excluyó a la población con riesgo habitual de osteoporosis. Un futuro estudio dirigido a una población multirracial y más parecida a la que atendemos habitualmente será necesario para confirmar este importante hallazgo.
Un punto destacable del trabajo es que los niveles plasmáticos de homocisteína son un factor predictor independiente de fracturas y no se relacionan con la densidad ósea $u$ otros factores como las caídas. Esta observación coincide con anteriores ${ }^{2}$ y difiere del trabajo de Framingham donde los niveles de Vitamina B12 se asociaron a bajos niveles de densidad mineral ósea. Sin embargo, en dicho estudio no se había valorado la homocisteínemia, pudiendo este ser un factor independiente del metabolismo de la vitamina B, dato de interés a confirmar ${ }^{3}$.

En décadas anteriores se demostró que el tratamiento de las deficiencias de vitamina $\mathrm{D}$ y vitamina $\mathrm{K}$ disminuía la incidencia de fracturas por fragilidad ósea ${ }^{4 \cdot 5}$. En esta línea de observación se inscribe este trabajo.

\section{Conclusión del comentador}

la comprobación de la importancia de las Vitaminas D, K y B en la salud ósea de la población con mayor riesgo de fracturas es un hallazgo de magnitud, pues el diagnóstico y la corrección de estas carencias es simple y económica.

Luisa Plantalech [ Coordinadora de la Sección Osteopatías Médicas. Servicio de Endocrinología, Metabolismo y Medicina Nuclear . Hospital Italiano de Buenos Aires ]

Plantalech L. El tratamiento con folato y mecobalamina previene las fracturas de cadera en pacientes con accidente cerebro vascular. Evid. Actual. Pract. Ambul. 2005;8:166. Comentado de: Effect of Folate and Mecobalamin on hip fracture in Patients with stroke. A randomized controlled trial. Sato Y, Honda Y, Iwamoto J. y col. JAMA. 2005, March, 293:1082-1088. PMID: 15741530

Referencias

1. Mc Lean RR, Jacques PF, Selhub J, Tucker KL, Samelson EJ, Broe KE, Hannan MT, Cuples LA, Kiel DP. Homocisteine as predictive factor for hip fracture in older persons. N Engl J Med. 2004:350:2089-90

2. Van Meurs JB, Dhonukshe-Rutten RA, Pluijm SM, van der Klift M, de Jonge R, Lindemans J, de Groot LC, Hofman A, Witteman JC, van Leeuwen JP, Breteler MM, Lips P, Pols HA, Uitterlinden AG. Homocysteine levels and the risk of osteoprosisis fracture. N Engl J Med. 2004; 350: 2033-41.

3. Tucker KL, Hannan MT, Qiao N, Jacques PF, Selhub J, Cupples LA, Kiel DP. J Bone Min Res. 2005; 20:152-8.

4. Chapuy MC, Arlot ME, Duboeuf F, Brun J, Crouzet B, Arnaud S, Delmas PD, Meunier PJ. Vitamin D3 and calcium to prevent hip fractures in the elderly women. N Engl J Med. 1992 $327: 1637-42$

5. Shiraki M, Shiraki Y, Aoki C et al. Vitamin K(menatrenone) effectively prevents fracture and sustains lumbar bone mineral density in osteoporosis. J Bone Min Res. 2000;15:515-21. 